\title{
Lessons from the elimination of poliomyelitis in Africa
}

\section{Abdulaziz Mohammed, Oyewale Tomori and John N. Nkengasong}

Abstract | In August 2020, Africa was declared free of poliomyelitis (polio), bringing to fruition a goal that took more than 30 years to achieve. This Perspective chronicles global, continental, national and community actions taken by diverse stakeholders that finally led to the elimination of transmission of wild poliovirus in Africa. The cascade of events started with the development of polio vaccines and the realization that polio, much like smallpox, could be eradicated. After a 1988 pledge by the World Health Assembly to eradicate polio globally, concerted and deliberate efforts were made in Africa to achieve this goal. This included the use of evidence-based approaches for the harmonization and standardization of public health strategies, using a network of polio laboratories and emergency operation centres and actively pursuing underserved populations. Innovative solutions to counter challenges such as conflict and vaccine hesitancy may be of use in future public health interventions.

Poliomyelitis (polio) is a viral disease that can cause lifelong paralysis in about $1 \%$ of all infected individuals (paralytic polio) and often starts in childhood. Archaeological evidence suggests that a disease that caused lameness in humans may have already existed in the ancient Egyptian civilization. However, not until in 1908 did two scientists provide evidence that polio, one of the major causes of lameness at the time, was caused by a viral agent ${ }^{1}$ (FIG. 1). Karl Landsteiner and Erwin Popper used scientific methods available at that time to prove that polio is caused by an infectious agent that is smaller than bacteria. In 1950, the virus was visualized for the first-time by electron microscopy ${ }^{2}$, and effective vaccines were subsequently produced - inactivated polio vaccine ${ }^{3}$ in 1955 , and attenuated polio vaccine or oral polio vaccine $(\mathrm{OPV})^{4}$ in 1961 (FIG. 1).

However, despite intensive efforts to control the disease globally, in 1988, it was estimated that 350,000 children were paralysed by polio ${ }^{2}$, with most cases occurring in African and Asian countries. In the same year, the World Health Assembly (WHA) pledged to eradicate polio globally by 2000 and initiated the Global Polio Eradication Initiative (GPEI). Although Africa and the world missed this target, in 2020, right in the middle of a global COVID-19 pandemic, Africa was certified as polio-free. Although it took 32 years to eliminate polio in Africa, the achievement constitutes a giant leap for worldwide efforts to eradicate the disease. Presently, only Pakistan and Afghanistan have endemic transmission of wild poliovirus (WPV). WPV consist of three serotypes type 1 , type 2 and type 3 - and owing to difference in constituent proteins, there is no cross-protective immunity following exposure to one of the serotypes in the remaining two. Type 2 was eradicated in 2015 , type 3 was eradicated in 2019 and only type 1 remains in natural transmission in some parts of the world ${ }^{3}$. Here we discuss the challenges encountered during the elimination of polio in Africa, particularly in Nigeria, which was the last country in Africa to be certified polio-free. We discuss the lessons that can be learnt and their potential applicability to future public health interventions in Africa and elsewhere.

\section{Africa's journey to polio elimination}

In 1980, when smallpox was officially declared as eradicated, public health experts had gained valuable experience spanning a 20-year period on how to use a combination of vaccination, surveillance and other preventive measures to interrupt the natural transmission and ultimately eradicate a viral pathogen ${ }^{4}$. Around the same time, epidemiologists and researchers working on polio control programmes around the world, and especially in the World Health Organization (WHO) Region of the Americas, were beginning to gain a better understanding of the epidemiology of WPV and how a blend of routine immunization, mass vaccination campaigns and surveillance for acute flaccid paralysis (AFP), using a network of laboratories, could potentially replicate the smallpox success story for polio eradication.

In the early 1950s, researchers considered polio as a public health problem that affected mainly affluent nations with low infant mortality. Conversely, surveillance data were indicating that countries with high infant mortality, like most of Africa, had a lower incidence of polio, which was counter intuitive because of the strong correlation between high infant mortality and polio risk factors ${ }^{5,6}$. The erroneous belief could have been caused by the under-reporting of cases of polio in countries with high infant mortality rates due to non-standardized surveillance systems for polio ${ }^{6,7}$. However, a series of lameness surveys conducted in various countries on the African continent in the 1970s quickly dispelled the myth of a low burden of paralytic polio in Africa and revealed a high prevalence and incidence of the disease, and its association with poor sanitation, poor design of housing and other poverty-related social determinants of health (BOX 1). Motivated by the earlier success in polio elimination by countries such as Cuba, and by the eradication of smallpox, the WHA passed a resolution on the Expanded Programme on Immunization in $1974\left(\mathrm{REF}^{8}{ }^{8}\right)$. The goal of the resolution was for WHO member states to develop strategies for immunization and surveillance programmes against infectious disease such as polio, pertussis, measles, tuberculosis, tetanus and smallpox. This was closely followed by the Declaration of Alma-Ata in 1978, which identified primary health-care as crucial to the attainment of 'Health for All'. This declaration specifically listed immunization against major diseases and the control of endemic diseases as central elements of primary care ${ }^{9}$. 


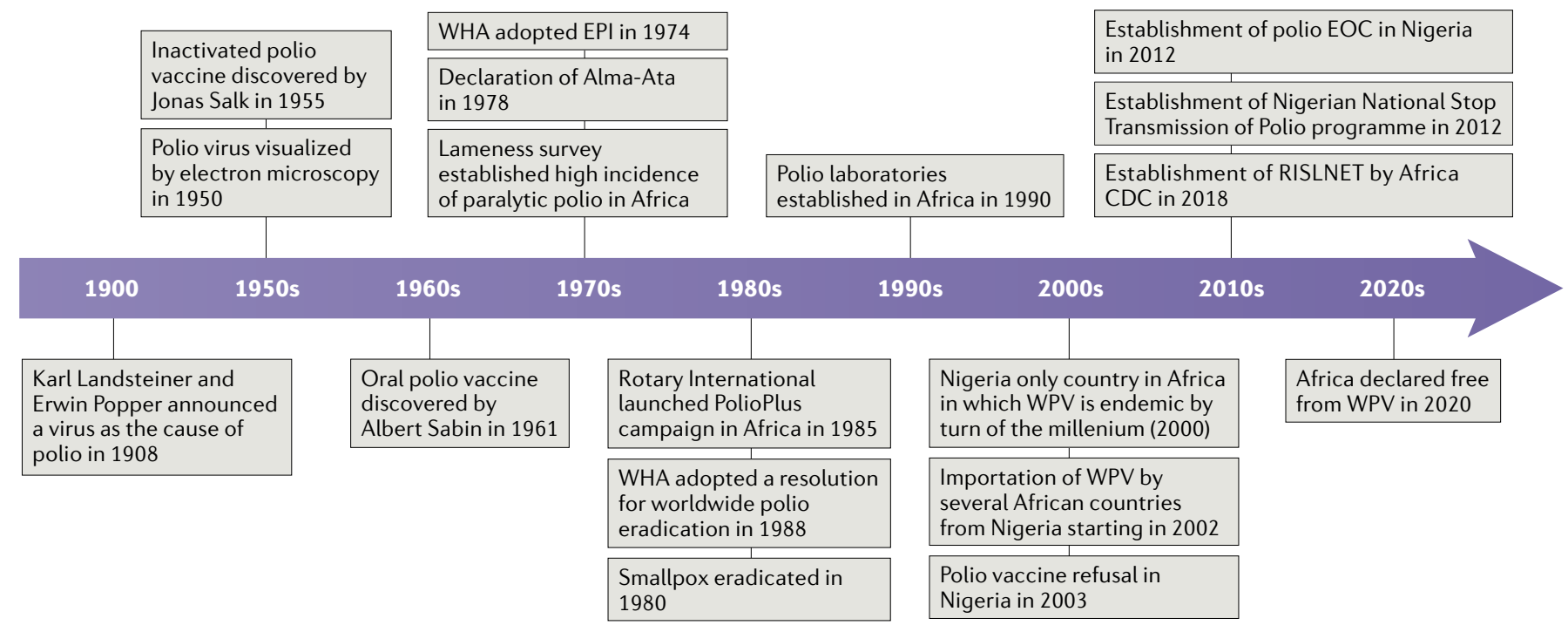

Fig. 1 | Timeline of major events on the journey to the certification of Africa as polio-free. Africa CDC, Africa Centres for Disease Control and Prevention; EOC, emergency operation centre; EPI, Expanded Programme on Immunization; RISLNET, Regional Integrated Surveillance and Laboratory Network; WHA, World Health Assembly; WPV, wild poliovirus.

As a result of childhood immunization campaigns and surveillance for paralytic polio, the WHO Region of the Americas was declared polio-free in 1994, the WHO Western Pacific Region in 2000, the WHO European Region in 2002 and the WHO South-East Asia Region in $2015\left(\mathrm{REF}^{8}\right)$. However, the success in the elimination of polio in the WHO Region of the Americas, Europe and some tropical Asian countries could not be replicated in Africa with the traditional methods of routine immunization and a few mass immunization campaigns. A review of vaccination against polio in economically underdeveloped countries found that a high prevalence of infection with enteric non-poliovirus (as observed in at-risk populations in Africa) lowers the frequency of seroconversion after one dose of immunization using trivalent OPV (TABLE 1), an obstacle that can be mitigated by repeated dosing ${ }^{6}$. To achieve and sustain herd immunity, which would allow the interruption of WPV transmission among susceptible hosts, widespread immunization with a minimum coverage of $90 \%$ of the susceptible population in Africa was required. This could be achieved only through immunization with trivalent OPV (TABLE 1) using multiple vaccination strategies, such as routine immunization and supplemental immunization activity, with the latter complementing routine immunization by targeting the members of the susceptible population who were missed or were only partially protected.
The specific challenges for the GPEI in

Africa. The GPEI was built on lessons learnt from earlier efforts to control polio in North America, Cuba, Brazil and some European countries, with strategic adjustments to meet the specific challenges facing many African and Asian countries. In 1985, partly inspired by the success of its support for the polio immunization campaign in the Philippines, Rotary International launched the PolioPlus campaign to rid the world of paralytic polio $^{10}$. In 1988, the WHA adopted a resolution for the worldwide eradication of polio ${ }^{11}$. The critical pillars identified for the eradication of polio were (1) high infant routine immunization coverage with four doses of trivalent OPV in the first year of life, (2) regular supplemental immunization activity during national immunization days to provide supplementary doses of OPV to all children younger than 5 years, as well as 'mop-up' campaigns that specifically target outbreaks in defined geographic areas, and (3) active case-based surveillance for WPV through reporting and laboratory testing of all cases of AFP among children younger than 15 years $^{12}$.

By the turn of the century, the GPEI was increasingly confident that the transmission of WPV would be interrupted in the African region. The confidence was based on the fact that a combination of these strategies had reduced cases of WPV by 99\% globally, and in Africa, only Nigeria remained endemic for WPV. However, events in Nigeria in 2003 greatly diminished the optimism.
Vaccine refusal in northern Nigeria. In 2003, a disagreement between the Nigerian federal government and a number of subnational governments and religious leaders in the northern part of the country over the safety of OPV led to some states placing a total ban on the use of the vaccine. The effect of this ban was felt beyond these states and across the northern part of the country as some religious leaders openly preached against the use of $\mathrm{OPV}^{13}$. Historically in northern Nigeria, WPV transmission was largely due to a combination of socio-economic determinants of health such as poor demand for and access to health services, low immunization coverage, few available skilled health workers, extreme poverty and low literacy. This was complicated by community resistance to immunization services $^{14}$. In a study of households that refused OPV during mass immunization campaigns in northern Nigeria in 2011, it was found that the heads of households had a lower education level, lacked knowledge of benefits of immunization and did not know the global nature of the polio eradication campaign compared with households accepting OPV ${ }^{15}$.

The consequence was immediate and devastating. Within a short time, the reported number of laboratory-confirmed polio cases in Nigeria quadrupled, and by the end of 2003, accounted for nearly half the total worldwide cases ${ }^{16}$. Furthermore, countries that had been free of polio were soon reporting fresh outbreaks. Between 2002 and 2011, WPV imports, primarily from Nigeria, led to the reestablishment 
of WPV transmission and outbreaks in 39 countries that had previously been certified as polio-free ${ }^{17}$.

To contain the fallout, the GPEI conducted one of the largest social mobilization campaigns in the history of public health in Africa. Starting in 2003, concerted efforts were made to gain the trust of the state governments and communities across northern Nigeria and recruit influential traditional and religious leaders as champions for the polio elimination activities $^{18}$. In 2008, the Majigi programme systematically engaged the traditional, religious and political leadership to promote acceptance of the polio vaccination programme in high-risk communities where WPV was known to circulate and where resistance to vaccination was strong ${ }^{19}$. UNICEF and partners supported thousands of voluntary community mobilizers in northern states of Nigeria to promote mass vaccination campaigns, child and maternal health-care routine immunization, messaging on handwashing with soap, exclusive breastfeeding, the prevention and treatment of diarrhoea and screening for malnutrition. These efforts included engagement of community influencers such as traditional and religious leaders to ensure community buy-in. This resulted in hundreds of thousands of households reversing their decision to refuse vaccination, thereby allowing millions of children younger than 5 years to received their first OPV vaccination, markedly reducing the population susceptible to $\mathrm{WPV}^{14,20}$. With the benefit of hindsight, we postulate that the GPEI should have made community mobilization and participation one of the main strategic pillars of polio eradication at the onset, much like the immunization and surveillance strategies.

\section{Conflicts and their effect on polio}

elimination. Almost from the onset of the GPEI until the time Africa became certified as polio-free, regional conflicts were a reoccurring challenge. Three active conflict zones were particularly challenging: the conflict in the Horn of Africa, which involved Somalia, Ethiopia and Sudan ${ }^{21}$, the conflict between the Democratic Republic of the Congo and neighbouring countries $^{22}$ and the conflict in the Lake Chad region where the borders of Nigeria, Chad, Cameroon and Niger converge. The conflict in the Lake Chad region constituted the single most challenging security situation for the Polio elimination campaign in Africa ${ }^{23-25}$. In February 2013, insurgents killed polio workers and destroyed health-care facilities in Kano State, the most populous state in Nigeria and one of the states with the highest risk of polio at the time ${ }^{26,27}$. The urgency of the situation was further heightened when, in August 2016, two cases of WPV were detected in the conflict zone after more than 2 years of no reported circulation of WPV. It was later determined that WPV circulation went undetected for 2 years owing to gaps in surveillance caused by inaccessible areas under the control of insurgents ${ }^{28,29}$. To address the challenges in the Lake Chad region, and especially in north-eastern Nigeria, the Nigerian government adopted several strategies that enabled the delivery of vaccines to the at-risk population and improved the surveillance for AFP. This included the deployment of permanent health teams that consisted of personnel from within the community, shorter periods of intervention from outside experts and vaccination campaigns at border crossing points $^{24}$. In addition, security personnel were engaged $^{30}$. The lessons learnt from the success of the strategies can be applied to similar conflict zones where public health intervention is contemplated.

\section{The challenge of mobile populations. One} consistent finding from stool samples that tested positive for WPV in some African countries was the significant number of cases among mobile populations, especially pastoralist populations of western and eastern Africa ${ }^{31}$. In Nigeria, of the 112 cases of WPV infection with a date of onset in 2012, $80 \%$ were found within or close to underserved populations of nomadic pastoralists ${ }^{32}$. This suggests that these populations were contributing to the transmission of polio in Nigeria and indicated that some of the sporadic cases in other countries that were genetically linked to cases in Nigeria might have been imported through nomadic pastoralists moving across western and central Africa. To specifically address this challenge, in July 2012, the Nigerian government, in collaboration with the Nigerian Field Epidemiology and Laboratory Training Program and the US Centers for Disease Control and Prevention, established the Nigerian National Stop Transmission of Polio (NSTOP) programme. One of its strategic objectives was to target underserved populations, with challenges including the remoteness of the settlements, the lack of adequate resources to support outreach teams and the frequent seasonal movements of nomads ${ }^{32,33}$. Over several years of targeted interventions, the NSTOP deployed hundreds of staff to many underserved locations in Nigeria and was able to detect hundreds of previously unreported cases of AFP in numerous settlements that had not previously been reached by polio vaccination campaigns. The NSTOP also had the strategic objective of strengthening the overall management of the polio eradication programme and employed targeted technical assistance for polio eradication activities and operational research, all of which brought dynamism and added impetus to the Nigerian polio eradication programme during a period of need.

\section{Box 1 | Lameness survey established high incidence of paralytic polio in Africa}

After incomplete surveillance data from the 1960s and 1970s suggested the incidence of paralytic polio in Africa was low, several lameness surveys provided evidence to the contrary. For example, a representative lameness survey of school-aged children in Ghana in 1977 found that the prevalence of lameness attributable to polio ranged between 5 and 8 per 1,000 school-aged children and found the mean annual incidence of paralytic polio to be 23 per 100,000 population ${ }^{45}$. A similar survey in Niger in 1982 identified a prevalence of lower limb motor disorders in school-aged children of 7 per 1,000, with an estimated incidence of 45 per 100,000 population ${ }^{51}$. In Cameroon, in 1983 , an incidence of lameness of 18.8-32.6 per 100,000 population was found ${ }^{52}$. A review of lameness surveys conducted in developing countries identified differences in methods and the interpretation of findings between the different studies. The following conclusions were drawn from the various lameness surveys conducted at the time.

- The rates of paralytic polio in some countries were similar to those of countries in the World Health Organization Region of the Americas in the prevaccination period.

- There was an exceedingly high level of under-reporting of polio cases in Africa.

- A high prevalence of polio occurs in combination with high infant mortality in some African countries.

- Most cases occurred in children younger than 5 years.

- A standardized case definition was required, which needed to include the use of laboratory confirmation.

- There was a need for targeted intervention using methods that had proven successful in American and European countries, with some modifications to suit the particular needs of tropical and subtropical regions. 


\section{The Global Polio Laboratory Network}

To achieve the objective of the GPEI in

Africa, there was an urgent need for a sensitive surveillance system for AFP to allow timely and targeted implementation of immunization-based strategies in highrisk populations. The AFP surveillance is based on identifying and reporting children with AFP, transferring stool samples for laboratory analysis using the reverse cold chain method and isolating and sequencing polioviruses from the samples ${ }^{34}$. At the start of the GPEI, this was a huge challenge, especially in Africa. To address this challenge, in 1990, the GPEI, led by the WHO and national governments, established the Global Polio Laboratory Network, which was tasked with distinguishing WPV as a cause of AFP from paralysis caused by other diseases. Since then, a uniform annual accreditation exercise has monitored the accuracy and quality of testing and laboratory processes ${ }^{35}$. The network consists of 145 laboratories structured into three tiers: national, regional reference and global specialized laboratories ${ }^{36}$. Several studies have validated the immense contribution of the African polio laboratories to achieving early detection of outbreaks of WPV in defined geographic areas $\mathrm{s}^{22,24,37,38}$. Specifically, the polio laboratories can be credited with groundbreaking work in strengthening the laboratory workforce on the continent, polio diagnostics and the introduction of technologies such as next-generation sequencing to study the molecular epidemiology of WPV to guide outbreak responses, quality assurance and allow proficiency testing. The laboratory network, in coordination with the AFP surveillance, ensured that all communities in Africa had access to the latest laboratory diagnostics for polio, including cutting-edge molecular diagnostic procedures. The polio laboratories in Africa played crucial roles in providing scientific evidence that was decisive in addressing challenges for polio elimination in Africa. In Nigeria, between 2000 and 2016, there was suspicion of suboptimal surveillance for AFP. This was confirmed by environmental sampling of WPV in sewage and other sources ${ }^{38,39}$ and by molecular epidemiology to trace imported cases of WPV in Chad, Sudan, Ethiopia ${ }^{40}$, Cameroon and Equatorial Guinea ${ }^{41}$ to Nigeria. Moreover, orphan WPV (defined as polioviruses in which nucleotides at $1.5 \%$ or more of positions differ from those of known poliovirus) was detected, indicating continued transmission of WPV over a long period without detection by the surveillance system $^{39}$.

The Global Polio Laboratory Network has therefore been critical in the elimination of polio in Africa; however, whether it can also aid in the timely detection of other emerging and re-emerging infectious diseases will need to be evaluated. Here, the WHO joint external evaluation (JEE) may be of use. The JEE helps countries to identify the most critical gaps within their human and animal health systems so as to prevent, detect and rapidly respond to public health risks such as emerging and re-emerging infectious diseases. A 2018 review of Africa JEE performance concluded that major gaps existed with regard to biosafety and biosecurity. In addition, there was a general suboptimal performance in the technical area of 'detection' ${ }^{42}$, a thematic area of the JEE that includes assessing the ability of laboratory systems on the continent to reliably detect outbreaks. Therefore, there is a need to build on the lessons learnt from the Global Polio Laboratory Network to strengthen and establish all hazard laboratory and surveillance networks in Africa. The COVID-19 pandemic clearly illustrates the urgency to ensure that critical public health assets and networks such as the Global Polio Laboratory Network are able to adapt quickly. In 2018, the Africa Centres
Table 1 | Oral polio vaccines

\begin{tabular}{lll}
$\begin{array}{l}\text { Oral polio } \\
\text { vaccine }\end{array}$ & Protection offered & Characteristics \\
\hline tOPV & Serotypes 1, 2 and 3 & $\begin{array}{l}\text { Inexpensive, effective and offers long-lasting } \\
\text { protection }\end{array}$ \\
\hline bOPV & Serotypes 1 and 3 & $\begin{array}{l}\text { Stronger immune response against types } 1 \text { and } 3 \\
\text { compared with tOPV. No immunity against serotype 2 }\end{array}$ \\
\hline mOPVs & $\begin{array}{l}\text { Serotype 1 (mOPV type 1), } \\
\text { serotype 2 (mOPV type 2) } \\
\text { and serotype 3 (mOPV type 3) }\end{array}$ & $\begin{array}{l}\text { Generate best immune response to the targeted } \\
\text { serotype }\end{array}$ \\
\hline nOPV2 & Serotype 2 & $\begin{array}{l}\text { Genetically stable and less likely to be associated with } \\
\text { the emergence of cVDPV2 in low-immunity settings }\end{array}$ \\
\hline
\end{tabular}

bOPV, bivalent oral polio vaccine; cVDPV2, circulating vaccine-derived poliovirus type 2; mOPV, monovalent oral polio vaccine; nOPV2, novel oral polio vaccine type 2; tOPV, trivalent oral polio vaccine. for Disease Control and Prevention (Africa CDC) established the Regional Integrated Surveillance and Laboratory Network. Its aim is to support African Union member states to network critical public health assets and resources to enhance responses to outbreaks and improve pandemic preparedness. The Regional Integrated Surveillance and Laboratory Network was piloted in central Africa to coordinate and integrate all public health laboratory, surveillance and emergency response assets, including public health data ${ }^{43}$. In the coming years, the Africa CDC will be strengthening this type of network in all African regions to support the prevention, rapid detection and response to current and emerging public health threats on the continent ${ }^{44,45}$.

\section{The role of polio emergency operation centres}

By 2012, Nigeria was the only African country with natural transmission of WPV. To support polio elimination in Africa, the GPEI began establishing polio emergency operation centres (EOCs) in Nigeria to coordinate human and material resources. The WHO defines an EOC as a physical location for the coordination of information and resources using the incident management system (IMS), which is defined as a system that guides all levels of government and other partner organizations in working together to prevent, protect against, mitigate, respond to and recover from incidents. EOCs often use the IMS structure to apply a common organizational model or framework to all levels of emergency management responsibility within a jurisdiction, from the national government to front-line emergency response services ${ }^{46}$. The EOC in Nigeria used the IMS structure to ensure a flexible arrangement that allowed all government agencies, international agencies, local non-governmental organizations and the private sector to work harmoniously together in the polio programme ${ }^{47}$. Therefore, under the leadership of a government-appointed incident manager, the EOC oversees the implementation of the incident action plan and ensures accountability for all health workers involved in the response to key polio control activities such as effective mass immunization campaigns, response to outbreaks in confirmed areas of WPV transmission or targeted 'mop-up campaigns'. Using a cluster arrangement, partner organizations were assigned well-defined roles that limited any duplication of efforts and allowed close supervision and accountability. Thus, the 
WHO was responsible for surveillance and epidemiology, UNICEF for vaccine logistics and social mobilization, the US Centers for Disease Control and Prevention for laboratory and risk mapping and the NSTOP for outreach to underserved and mobile populations. The polio EOC eventually impacted on the polio programme in Nigeria by ensuring that data-driven policies were used for real-time decision-making and by strengthening both national and partner accountability. At the onset of the 2014 Ebola virus disease (EVD) outbreak in Nigeria, the polio EOC structure had been operational for more than 2 years. Three days after the first case of EVD had been confirmed in Nigeria, the polio EOC quickly transformed into an EVD EOC in the commercial city of Lagos, where the first case was reported ${ }^{47}$. The IMS for this project was headed by the incident manager of the polio EOC. This ensured that expertise and lessons learnt from the polio EOC were readily available to the EVD EOC. Nigeria continues to use a multihazard EOC, now situated within the Nigeria Centre for Disease Control, for the response to subsequent public health events, including the COVID-19 pandemic. To further strengthen the capacity and capability of African Union member states in multihazard and multisectoral preparedness and response for public health emergencies at national, regional and continental levels, the Africa CDC is supporting the establishment of functional national public health EOCs in African Union member states.

Future considerations for the GPEI in Africa. In August 2020, after a rigorous 4-year certification process, Africa celebrated being declared free of endemic transmission of all the three types of WPV. However, the existence of conflict zones and hard-to-reach areas with low routine immunization coverage meant that a rare form of the infection, a vaccine-derived version of the poliovirus, continues to affect some African countries ${ }^{48}$. Of the 31 active outbreaks of circulating vaccine-derived poliovirus type 2 globally from July 2019 to February 2020, 24 (77\%) occurred in African countries, with 7 of these spreading internationally (BOX 2).

To control this outbreak, African countries need to increase routine immunization coverage using bivalent OPV and injectable inactivated polio vaccine, while at the same time improving the quality of supplemental immunization activity using monovalent $\mathrm{OPV}^{49}$. It is expected that African countries with active outbreaks

\section{Box 2 | Circulating vaccine-derived poliovirus}

On extremely rare occasions, an excreted vaccine-derived virus can change genetically into a form that can be transmitted and cause paralysis. This is referred to as "circulating vaccine-derived poliovirus' (cVDPV). Generally, the strain will have been circulating in an unimmunized or underimmunized population for at least 12 months before the genetic change. Therefore, cVDPVs occur only when routine or supplementary immunization activities are poorly conducted and a population is left susceptible to poliovirus. Vaccine-derived polioviruses are not a risk of the vaccine itself, but are a risk as a result of low vaccination coverage as fully immunized populations are protected against both vaccine-derived and wild polioviruses. The small risk of the occurrence of $\mathrm{cVDPVs}$ is measured against the tremendous public health benefits associated with oral polio vaccine. Over the past 20 years since widespread administration of oral polio vaccine started, more than ten million cases of polio have been averted ${ }^{53}$.

of circulating vaccine-derived poliovirus type 2 will have access to novel OPV type 2 that was granted WHO Emergency Use Listing recommendation in November 2020 (REF. ${ }^{50}$ ). Novel OPV type 2 has been shown to be safe and effective and more genetically stable than monovalent OPV type 2 (TABLE 1), ensuring that it is less likely to change into a form that causes paralysis in African communities with low routine immunization coverage.

\section{Conclusion}

In August 2020, on the occasion of certification of Africa as polio-free, WHO Director-General Tedros Adhanom Ghebreyesus said "ending wild poliovirus in Africa is one of the greatest public health achievements of our time and provides powerful inspiration for all of us to finish the job of eradicating polio globally" 44 . The importance of stakeholder engagement and ensuring community mobilization as well as creativity to surmount challenges such as vaccination campaigns for nomadic underserved communities as well as in conflict zones are important lessons learnt from the African experience. Undoubtedly, the continent has made tremendous gains in the use of central coordination of human and material resources, together with effective use of laboratories for diagnosis and mechanisms of surveillance to inform public health outbreak responses as outlined hereon. However, more needs to be done to ensure that these achievements continue to serve the continent beyond the GPEI intervention. This can be achieved only by supporting African Union member states to develop strong national public health institutions that can bring all the lessons learnt from the global eradication initiative under one strong public health leadership. The Africa CDC is already supporting national public health institutions across African Union member states to create strong institutions that can coordinate core public health functions of surveillance, laboratory networking, workforce development and management of EOCs to safeguard the health security of the residents of Africa.

Abdulaziz Mohammed ${ }^{1}$, Oyewale Tomori ${ }^{2}$ and John N. Nkengasong (iD 1 凶

${ }^{\prime}$ Africa Centres for Disease Control and Prevention, Addis Ababa, Ethiopia.

${ }^{2}$ Redeemer's University, Ede, Nigeria.

凶e-mail: nkengasongJ@africa-union.org https://doi.org/10.1038/s41577-021-00640-w Published online 25 October 2021

1. College of Physicians Philadelphia. The History of vaccines https://www historyofvaccines org/content/ poliovirus-identified (2021).

2. World Health Organization. WHO Africa: Health Topics: Polio https://www.afro.who.int/health-topics/ polio (2020).

3. Global Polio Eradication Initiative. The virus https:// polioeradication.org/polio-today/polio-prevention/ the-virus/ (2021)

4. World Health Organization. Smallpox Eradication Programme-SEP (1966-1980) https://www.who.int/ news-room/feature-stories/detail/the-smallpoxeradication-programme---sep-(1966-1980) (2010).

5. Viguera Ester, P., Torres, A., Freire, J. M., Hernández, V. $\S$ Gil, Á. Factors associated to infant mortality in sub-Saharan Africa. J. Public Health Africa 2, 57 (2011).

6. Sabin, A. B. Vaccination against poliomyelitis in economically underdeveloped countries. Bull. World Health Org. 58, 141-157 (1980).

7. Hampton, L. Albert Sabin and the coalition to eliminate polio from the Americas. Am. J. Public Health 99, 34-44 (2009)

8. World Health Organization. Poliomyelitis https://www. who.int/news-room/fact-sheets/detail/poliomyelitis (2019).

9. World Health Organization. WHO called to return to the Declaration of Alma-Ata https://www.who.int teams/social-determinants-of-health/declarationof-alma-ata (2015)

10. Sever, J. L. et al. Rotary's PolioPlus Program: lessons learned, transition planning, and legacy. J. Infect Dis. 216 (suppl. 1), S355-S361 (2017).

11. Global Polio Eradication Initiative. History of polio http://polioeradication.org/polio-today/history-of-polio/ (2020).

12. US CDC. CDC and the Global Polio Eradication Initiative https://www.cdc.gov/polio/who/index.htm (2013).

13. Obadare, E. A crisis of trust: history, politics, religion and the polio controversy in Northern Nigeria. Patterns Prejudice 39, 265-284 (2005).

14. Duru, J. I., Usman, S., Adeosun, O., Stamidis, K. V. $\&$ Bologna, L. Contributions of volunteer community mobilizers to polio eradication in Nigeria: the experiences of non-governmental and civil society organizations. Am. J. Trop. Med. Hygiene 101, 74-84 (2019).

15. Mohammed, A. et al. Characteristics of persons refusing oral polio vaccine during the immunization plus days-Sokoto, Nigeria 2011. Pan African Med. J. 18, 10 (2014) 
16. Chen, C. Rebellion against the polio vaccine in Nigeria implications for humanitarian policy. African Health SCi. 4, 205-207 (2004)

17. Michael, C. A. et al. Polio legacy in action: using the polio eradication infrastructure for measles elimination in Nigeria - the National Stop Transmission of Polio Program. J. Infect. Dis. 216, 373-379 (2017).

18. World Health Organization. Kano State Nigeria, fully endorses OPV https://www. afro.who.int/news kano-state-nigeria-fully-endorses-opv (2004)

19. Nasiru, S.-G. et al. Breaking community barriers to polio vaccination in northern Nigeria: the impact of a grass roots mobilization campaign (Majigi). Pathogens Global Health 106, 166-171 (2012).

20. GPEI. Expanding social mobilization approaches to protect every last child http://polioeradication.org/ news-post/expanding-social-mobilizationapproaches-to-protect-every-last-child/ (2017).

21. UNICEF. Despite conflict, Horn of Africa one step from polio-free status https://reliefweb.int/report/ethiopia/ despite-conflict-horn-africa-one-step-polio-free-status (2002).

22. Deressa, W. et al. Lessons learned from the polio eradication initiative in the Democratic Republic of Congo and Ethiopia: analysis of implementation barriers and strategies. BMC Public Health 20, 1807 (2020).

23. Global Polio Eradication Initiative. "These places prove our biggest challenge" https://polioeradication.org/ news-post/these-places-prove-our-biggest-challenge/ (2018).

24. Nasir, U. N. et al. Polio elimination in Nigeria: a review. Hum. Vaccin. Immunother 12, 658-663 (2016).

25. Haddison, E. C., Ngono, D., Kouamen, G. T. \& Kagina, B. M. Successful polio supplementary immunisation activities in a security compromised zone - experiences from the southwest region of Cameroon. Vaccine. 36, 6961-6967 (2018 Nov)

26. BBC. Nigeria polio vaccinators shot dead in Kano https://www.bbc.com/news/world-africa-21381773 (2013)

27. Usman, S., Bologna, L. \& Stamidis, K. V. The CORE Group Partners Project in north east Nigeria: community engagement strategies to combat skepticism and build trust for vaccine acceptance. Am. J. Trop. Med. Hygiene 101, 68-73 (2019).

28. Khan, F. et al. Progress toward polio eradication worldwide, January 2016-March 2018. MMWR 67 524-528 (2018).

29. Higgins, J. et al. Finding inhabited settlements and tracking vaccination progress: the application of satellite imagery analysis to guide the immunization response to confirmation of previously-undetected, ongoing endemic wild poliovirus transmission in Borno State, Nigeri. Int. J. Health Geograph. 18, 11 (2019).

30. Nkwogu, L. et al. Impact of engaging security personnel on access and polio immunization outcomes in security-inaccessible areas in Borno state, Nigeria. BMC Public Health 18, 1311 (2018).

31. Haydarov, R. et al. Evidence-based engagement of the Somali pastoralists of the Horn of Africa in polio immunization: overview of tracking, cross-border, operations, and communication strategies. Global Health Commun. 2, 11-18 (2016).

32. Gidado, S. O. et al. Outreach to underserved communities in northern Nigeria, 2012-2013. J. Infect. Dis. 210, s118-s124 (2014).

33. Waziri, N. E. et al. Polio eradication in Nigeria and the role of the national stop transmission of polio program 2012-2013. J. Infect. Dis. 210, 111-117 (2014).

34. Craig, A. S. et al. The public health legacy of polio eradication in Africa. J. Infect. Dis. 216, 343-350 (2017).

35. Global Polio Eradication Initiative. The Global Polio Laboratory Network http://polioeradication.org/ polio-today/polio-now/surveillance-indicators/theglobal-polio-laboratory-network-gpln/ (2020).

36. Gumede, N. et al. Polio Eradication Initiative (PEI) contribution in strengthening public health laboratories systems in the African region. Vaccine 34 5164-5169 (2016)

37. Diop, O. M., Kew, O. M., de Gourville, E. M. \& Pallansch, M. A. The Global Polio Laboratory Network as a platform for the viral vaccine-preventable and emerging diseases laboratory networks. J. Infect. Dis 216, S299-S307 (2017).

38. World Health Organization. Report of the 23rd Informa Consultation of the Global Polio Laboratory Network (GPLN) https://polioeradication.org/wp-content/ uploads/2017/08/GPLN_Meeting_recommendations_ 2017.pdf (2017)

39. Asghar, H. et al. Environmental surveillance for polioviruses in the global polio eradication initiative. J. Infect. Dis. 210, S294-S303 (2014).

40. Tegegne A. A. et al. Characteristics of wild polio virus outbreak investigation and response in Ethiopia in 2013-2014: implications for prevention of outbreaks due to importations. BMC Infect. Dis. 18, 9 (2018).

41. Hagen J. E. et al. Progress toward polio eradication worldwide, 2014-2015. MMWR https://www.cdc. gov/ mmwr/preview/mmwrhtml/mm6419a5.htm (2015).

42. Talisuna, A. et al. Joint external evaluation of the International Health Regulation (2005) capacities: current status and lessons learnt in the WHO African region. BMJ Glob. Health 4, e001312 (2019).

43. Africa CDC. Regional Integrated Surveillance and Laboratory Network https://africacdc.org/rislnet/ (2020).

44. World Health Organization. Global Polio Eradication Initiative applauds WHO African Region for wild poliofree certification https://www.who.int/news/item/2508-2020-global-polio-eradication-initiative-applaudswho-african-region-for-wild-polio-free-certification (2020).
45. Ofosu-Amaah, S., Kratzer, J. H. \& Nicholas, D. D. Is poliomyelitis a serious problem in developing countries? - lameness in Ghanaian schools. British Med. J. 1, 1012-1014 (1977)

46. World Health Organization. Framework for a public health emergency operations centre November 2015 https://www.who.int/publications/i/item/frameworkfor-a-public-health-emergency-operations-centre (2020).

47. Vaz, R. G. et al. The role of the polio program infrastructure in response to Ebola virus disease outbreak in Nigeria 2014. J. Infect. Dis. 213 S140-S146 (2016).

48. World Health Organization. Polio https://www. afro. who.int/health-topics/polio (2020).

49. Global Polio Eradication Initiative. Outbreak preparedness $\&$ response: strategy for response to cVDPV2 2019-2021 https://polioeradication.org/ wp-content/uploads/2021/03/GPEl-cVDPV2-nOPV2Factsheet-20210312-EN.pdf (2019).

50. Global Polio Eradication Initiative. Novel oral polio vaccine type 2 (nOPV2) granted EUL recommendation https://polioeradication.org/news-post/novel-oral-poliovaccine-type-2-nopv2-granted-interim-emergency use-listing-recommendation/ (2020).

51. Thuriaux, M. C. A prevalence survey of lower limb motor disorders in school-age children in Niger and an estimation of poliomyelitis incidence. Trop. Geograph. Med. 34, 163-168 (1982).

52. Heymann, D. L., Floyd, V. D., Lichnevski, M., Maben, G. K. \& Mvongo, F. Estimation of incidence of poliomyelitis by three survey methods in different regions of the United Republic of Cameroon. Bull. World Health Org. 61, 501-507 (1983).

53. World Health Organization. Poliomyelitis: vaccine derived polio https://www.who.int/news-room/ q-a-detail/poliomyelitis-vaccine-derived-polio (2017).

\section{Author contributions}

A.M. researched data for the article. J.N.N. and O.T. contributed substantially to discussion of the content. A.M. and J.N.N wrote the article. All authors reviewed and/or edited the manuscript before submission.

\section{Competing interests}

The authors declare no competing interests.

\section{Peer review information}

Nature Reviews Immunology thanks Ananda Bandyopadhyay, and the other, anonymous, reviewer(s) for their contribution to the peer review of this work.

Publisher's note

Springer Nature remains neutral with regard to jurisdictional claims in published maps and institutional affiliations.

C) Springer Nature Limited 2021 